\title{
Impact of Scheduling Algorithms for QoS Improvement in LTE Networks
}

\author{
Chintalapati Rahul Varma, Ravikumar
}

\begin{abstract}
Spectral efficiency and delay are important design metrics that should be taken into consideration while designing a LTE/LTE-A network. Various factors effect these metrics in various ways. In this paper we will be analyzing spectral efficiency vs number of users and delay vs number of users in a 4G LTE network. Additionally, we will be analyzing effect of different scheduling types on delay and throughput of the network. The scheduling types we compare are maximum throughput, proportional fair and round robin. We will conclude on a scheduling type that gives us the least delay and maximum throughput. In view of the Universal Mobile Telecommunications System (UMTS) Long Term Evolution (LTE) technology, this paper considers multiple input multiple output (MIMO) capacity enhancement.
\end{abstract}

Index Terms: 4G, LTE, LTE-A, Scheduling types, Maximum Throughput, proportional fair, round-robin, spectral efficiency, delay.

\section{INTRODUCTION}

As 4-G wireless mobile networks, LTE-A (i.e. long-term development) and System Architecture Evolution combine. SAE is an improvement and improvement of the GPRS network, with the following changes as shown below [1]: Simpler mechanisms with smaller components in the core network, Significant increase in 3rd generation system throughput. Support and quality between different access networks that are not homogeneous.We will split the whole network into three main parts from figure one. They are Radio-Access, Evolved-Packet-Core

(RAN)Radio-Access-Network: it provides wireless wide-area mobile connectivity and is taken into account as a base station (BS) group. The base station lists all the carnal collective frequency transmissions carried out by the mobile (EPC) Evolved-Packet-Core: It can be regarded as SAE's fully IP-based flat principal. The EPC is the GPRS system correspondent and includes the Quality Management Object, the Helping portal, and the Packet Data Network gateway.The MME's primary function is to direct safety tasks such as authentication, clearance, and signing of Non-Access Stratum. It also controls idle condition value processing, roaming, and handover.

Revised Manuscript Received on October 30, 2019.

* Correspondence Author

Rahul Varma*, Department of Electronics and Communication Engineering, Vellore Institute Of Technology, VELLORE University, India.

Ravi Kumar CV, Assistant Professor, Department of Digital Electronics and Communication Systems, Jawaharlal Nehru Technological University, Ananthapur, India.

(C) The Authors. Published by Blue Eyes Intelligence Engineering and Sciences Publication (BEIESP). This is an open access article under the CC-BY-NC-ND license http://creativecommons.org/licenses/by-nc-nd/4.0/
Gateway Serving (S-GW) is connected with an UE and handles many objectives such as packet steering and forwarding.Home-Subscriber-Server could be a fundamental record that comprises user and grant-related data. There are many tasks that HSS manage like mobility organization [2], call and session establishment backing, user validation and entree endorsement

\section{RELATED WORKS}

In [2,3], they, in brief described how novel simulation instrument is authenticated to prototype LTE-A technology in addition as to assume several eminent algorithms for LTE-A. The most recent outcomes of this tool, in contrast to different papers, verifies that this simulation instrument is functioning as projected. constructed on this work, any idea for developing a pristine algorithm for LTE-A may be applied and achieved by this instrument. Its performance could be assessed and compared with existing algorithms [3,4]. Finally, with 3 algorithms being verified by 2 kinds of schedulers, the simulation outcomes verify that PF by means of Cross-CC is that the optimum algorithms for LTE-A. It will offer functional system throughput and finest fairness for all users together with "LTE, LTE-Advanced, and cell-edge" users. In [3,4], valuations of 3 basic packet scheduling algorithms (MR, PF, and RR) for the downlink of 3GPP LTE structures are thought-about. As a podium meant for analysis of these procedures, a organization parallel reformation of the LTE was established using MATLAB software. The evaluation amongst the procedures is done beneath numerous channel attenuation models. Simulation results illustrated that the MR algorithm achieved higher most rate, whereas RR provides most fairness among UEs. The PF scheduling technique, on the other hand, attained a decent balance amongst the throughput and also the equality among UEs.In [1], It is highly essential to choose a appropriate LTE transmission scheduler as we would like to satisfy inconsistent user throughput and resource impartiality wishes. This document accounts for the outcomes of a simulation study of 3 renowned uplink scheduling algorithms conducted to gage the procedure,. We tend to use the "LTE-Sim" open-source simulator with a mixed traffic stream generated by each user ("with 3 Best Exertions, 1 VoIP, and one Video"). Former analytical work focused mainly on estimating and reviewing all of these schedulers ' throughputs. This paper calculates the system execution of a number of well-known downlink schedulers in terms of output and hence the impartiality in terms of radio funding. The model results indicate a trade-off between the output and the index of fairness. 


\section{Impact of Scheduling Algorithms for QoS Improvement in LTE Networks}

In today's systems [4,5], Users are linked to base stations with the toughest signal intensity and the proportional fairness scheduling algorithm is carried out autonomously by each base station. This technique can lead in the best allocation of non-Pareto bandwidth while viewing the whole scheme.

Therefore, we formulate and study a comprehensive comparative fairness conundrum where user connotations to base stations are built on augmenting a comprehensive relational fairness goal [5]. We display that this hinderance is NP-hard and tough to estimate overall. Our outcomes illustrate that the throughput and fairness can both be enhanced in diverse user circulations when related to a method that allocates a operator to a base station with greatest signal intensity.

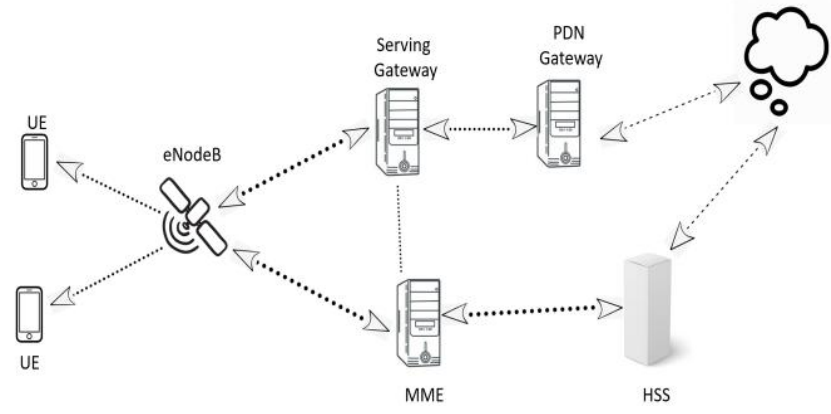

"Figure 1: Block diagram of a 4G-Lte network

\section{METHODOLOGY}

Maximum Throughput (MT) Scheduling type:

The algorithm employed in Max-Throughput scheduler is Scheduler maximizes throughput by continuously transferring RB's to the UE, which could potentially maximize overall throughput within the current TTI interval. It is primarily concerned about the CQI value to help UEs. Accordingly, UE with the best value of CQI will be assisted with the selected $\mathrm{RBs}$ and can therefore improve the overall performance of the selected cell[1]. However, its fairness value is not decent because, like cell-edge consumers, it does not negotiate enough belongings with destitute CQI norms for the UEs. These UEs can harm the truncated performance that can influence the cause of starvation.

First-Maximum-Expansion Scheduler (FME):

First-Maximum-Expansion Scheduler is especially anxious about capitalizing on the presentation of throughput and fairness. the chief initiative of F-M-E is utilizing the RB metrics that are dependent on assignment of a supply block (RB) to operator A with the most operative network state, then develop in mutual directions of the RB until the network upholds its best state midst diverse handlers. Once a user B with higher network state is discovered, user A is deemed aided and no additional chunks are going to be prearranged to it. The approximation complexness of the F-M-E scheduler [1] is $\mathrm{O}\left[\mathrm{N}^{*} \mathrm{NRB}\right]$, where $\mathrm{N}$ is the quantity of UE's and NRB is the total amount of accessible RB's within the network

Round Robin (R-R) Scheduling type:

This scheduler is network unaware, and it tries to allocate and assign RBs similarly and equitably between UEs[1]. the general throughput is extremely little associated to alternative algorithms. It additionally causes decrease within the efficacy of capitals since all UEs might not have alike QoS demand, in over-all, although the RR tries to allocate RBs similarly to them. using a trivial cell without interference condition can lead to having high rate fairness for RR related with different schedulers. Thus, the approximation of its complication is often stated with $\mathrm{O}(1)$.

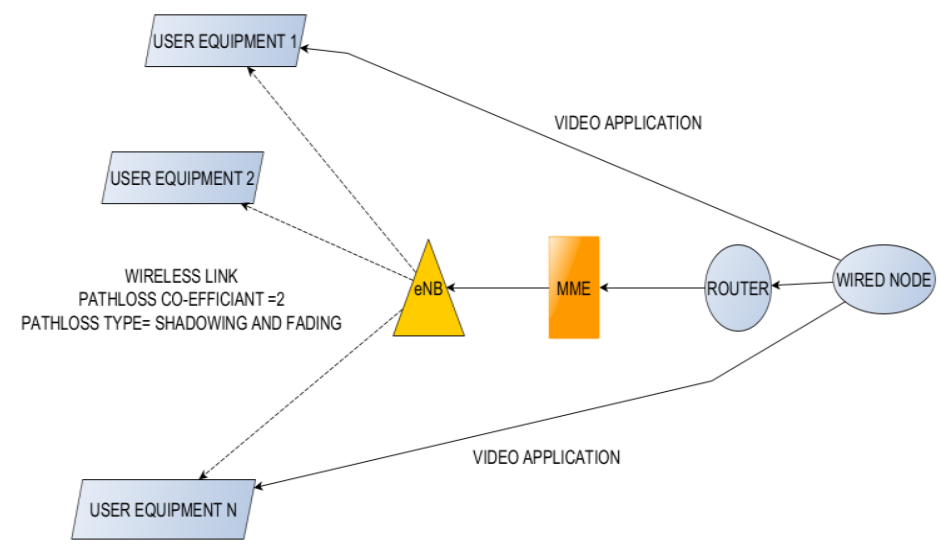

Figure 2: Block Diagram of the system model

Figure 2 is a system model of a $4 \mathrm{G}$ network consisting of a wired node, a router, a MME, an eNB and n- user equipment. The wired node, router, MME and eNB are wired connections. The connection between eNB and User equipment (UE) is established wirelessly. The parameters assumed while performing this experiment are mentioned in the table 1 below.

Formula 1 was used to measure the pathloss of the network. Formula 2,3,4 were used to find out the spectral efficiency of the network.

Table 1: Parameters used in experiment.

\begin{tabular}{|l|l|l|}
\hline & \multicolumn{1}{|c|}{ Parameter } & \multicolumn{1}{|c|}{ Value } \\
\hline 1. & Wired node Property & TCP+UDP \\
\hline 2. & Congestion control Algorithm & OLD HANOE \\
\hline 3. & Routing Protocol & OSPF \\
\hline 4. & Scheduling type & $\begin{array}{l}\text { Round-robin, } \\
\text { Proportional } \\
\text { fair, Max Th }\end{array}$ \\
\hline 5. & Transmission mode index & 1 \\
\hline 6. & Carrier aggregation & $\begin{array}{l}\text { Inter band non } \\
\text { contagious }\end{array}$ \\
\hline 7. & Channel bandwidth & $20 \mathrm{MHz}$ \\
\hline 8. & UE velocity & 0 \\
\hline 9 & No of users & $3,5,10,30,50,70$ \\
\hline 10 & Path loss & $\begin{array}{l}\text { Shadowing and } \\
\text { fading }\end{array}$ \\
\hline 11 & Path loss exponent & 2 \\
\hline
\end{tabular}

Formulas used:

$\bar{P}_{\text {Log }}=\ln \frac{\overline{\bar{P}}}{\overline{\bar{P}}}$ 


$$
f_{\bar{p}}(\bar{p})=\frac{1}{\sqrt{2 \pi \sigma \bar{p}}} \exp \left\{-\frac{1}{2 \sigma^{2}} \ln ^{2}\left(\frac{\bar{p}}{\overline{\bar{p}}}\right)\right\}
$$

$\frac{R_{b}}{B}=\frac{\log _{2} M}{\left(\operatorname{erfc} c^{-1}\left(P_{b, \text { target }}\right)\right)^{2}} \sin ^{2}\left(\frac{\pi}{M}\right) \frac{S}{N} \quad$ for $M-P S K$

$$
\frac{R_{b}}{R}=\frac{3 \log _{2} M}{2(M-1)\left(\operatorname{erfc}^{-1}\left(\frac{P_{b, \text { target } \log _{2} M}}{2}\right)\right)} \frac{S}{N} \quad \text { FOR } M-Q A M
$$

\section{RESULTS AND DISCUSSION:}

In this paper we run simulations on various parameters used in a $4 \mathrm{G}$ network. First, we plotted the values of spectral efficiency versus number of users. We achieved a maximum value of around $16 \%$ at 30 users. As we number of users increases the spectral efficiency starts to decrease as observed in figure 3. From figure 4, we can conclude that as the number of users are increasing the latency also increases in a linearly. From this figure we can also conclude that the rise in delay from 10 users to 30 users is greater that rise in delay from 30 to 50 users. From this we can conclude that as the number of users increases relative delay in the network decreases.

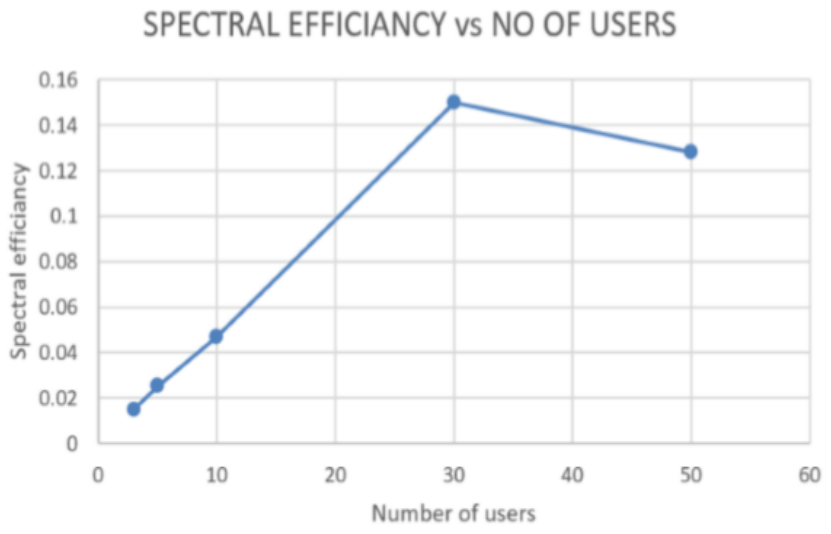

Figure-3: Spectral Efficiency vs no. of users (Table-4)

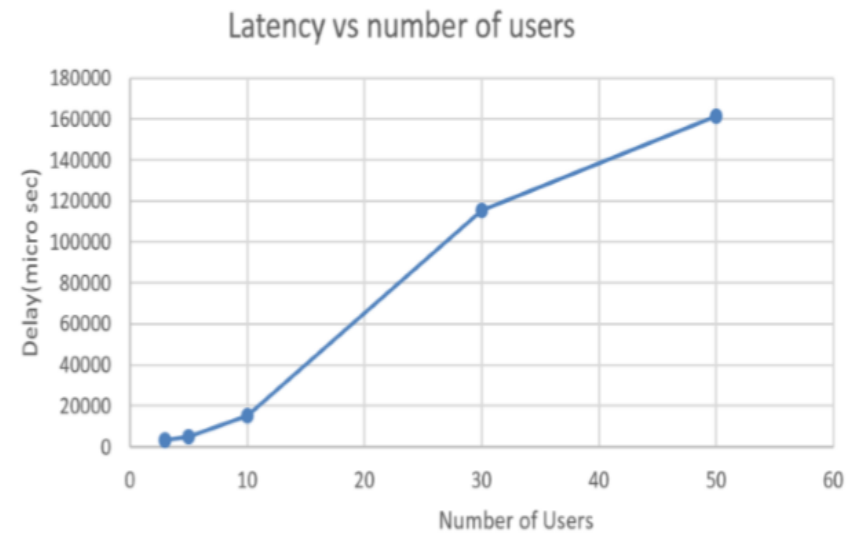

Figure-4: Latency Versus Number of Users (Table-5)

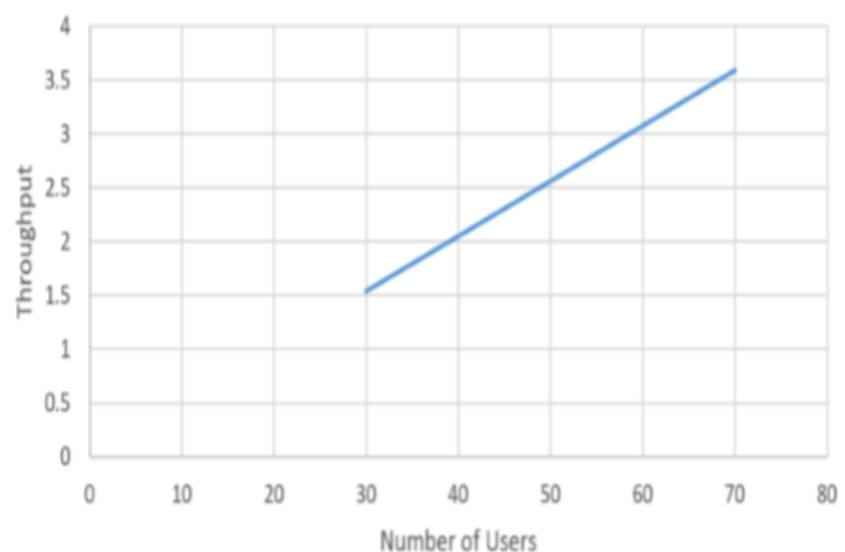

Figure-5: Variation of Throughput WRT number of users in maximum throughput scheduling

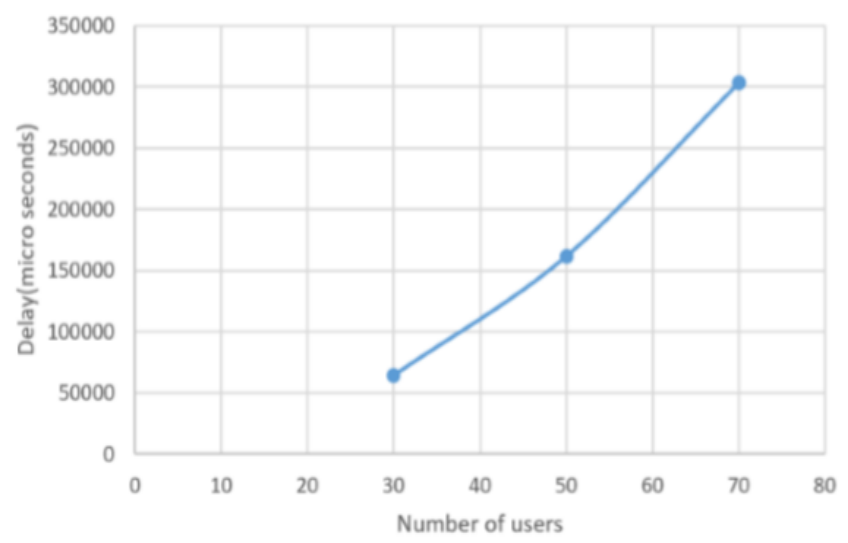

Figure-6: Delay (in Microsec) vesuss number of users In Maximum Throughput Scheduling

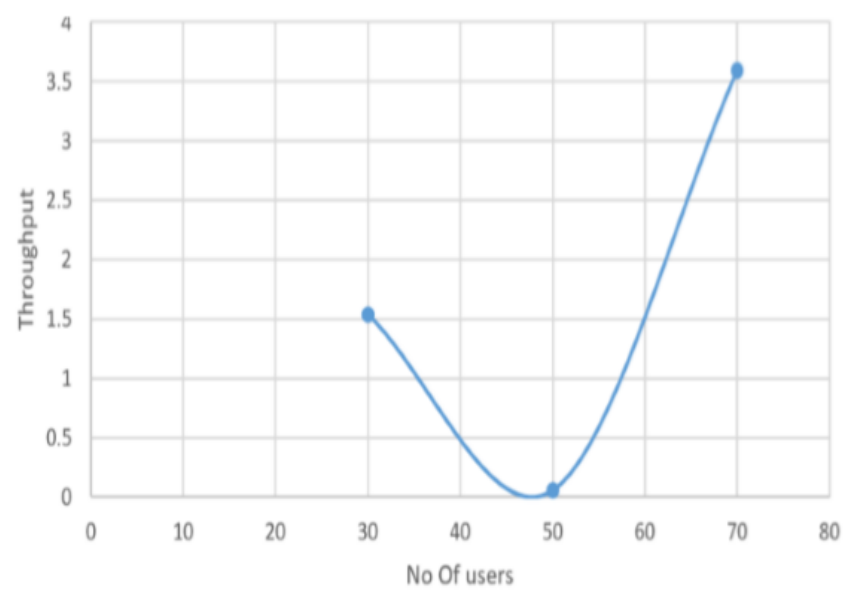

Figure-7: variation of Throughput with resp to no. of users in proportional fair scheduling 


\section{Impact of Scheduling Algorithms for QoS Improvement in LTE Networks}

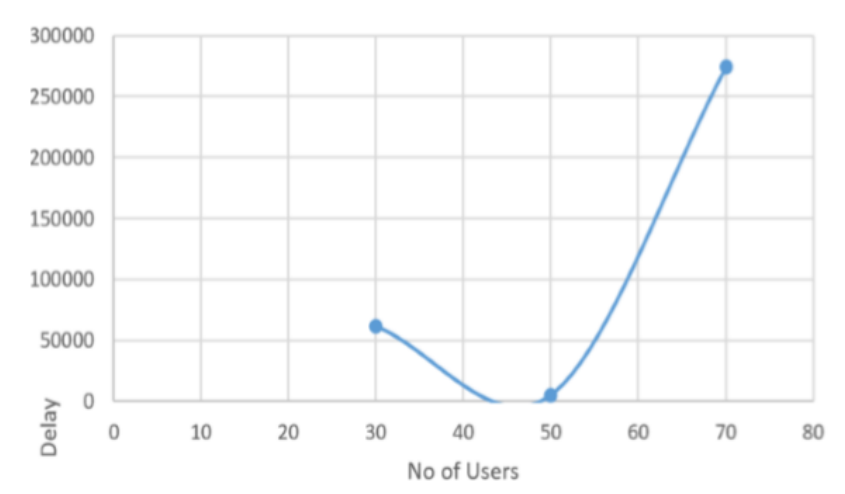

Figure-8: Delay (in Microsec) versus no. of users In PFS

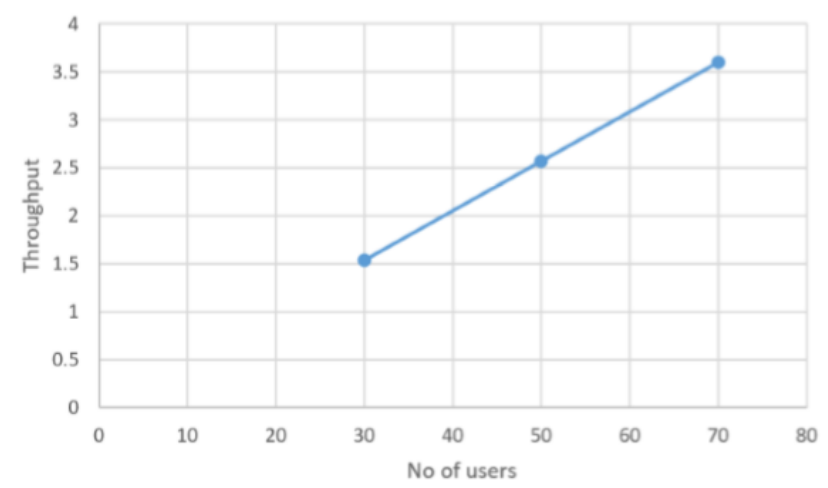

Figure-9: Throughput versus no. of users in RRS

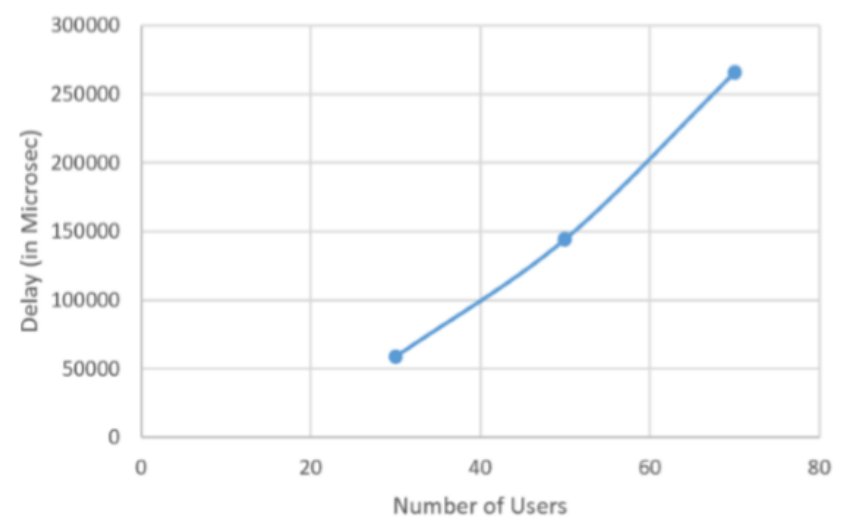

Figure-10: Delay (in Microsec) versus no. of users in RRS

In figure 5 , throughput in max throughput scheduling varies linearly with number of users until a point where it goes to a maximum value and then begins to drop.

In figure 6, we compare the trend in delay as we increase the number of users. The graph rises almost linearly and will continue to rise as the number of users increase until a point where the delay will obtain a value which is not feasible in practical applications. In figure 7, We will follow the trend in throughput with a proportional fair scheduling rise in user quantities. From the above graph we can observe that there is a sudden drop in throughput at around 50 users and they rises again. In figure 8 , we plot a graph between the delay and number of users. Clearly, we can observe that delay also suddenly at around 50 users and rises again. Thus in proportional fair scheduling, both the throughput and delay have a sudden fall before they start to rise again.

From Figure 9 we can observe that throughput vs number of users linearly increases for round robin scheduling. In figure
10 , With the amount of customers, the plot delay versus amount of users rises linearly.

From figure 11, We can compare delay in different scheduling types for different number of users. We can infer for the above graph that round robin scheduling type has the least amount of delay for all the different number of users we compared. Thus round robin scheduling can be implemented if we want our LTE- network to have the least delay.

From figure 12, we can infer that as number of users in a LTE network increases the throughput increases. By comparing the different scheduling types from above histogram, we can infer that Round robin scheduling gives the maximum throughput. From the above obtained inferences, we can arrive at a conclusion that Round Robin scheduling type for an LTE network gives the maximum throughput and minimum delay.

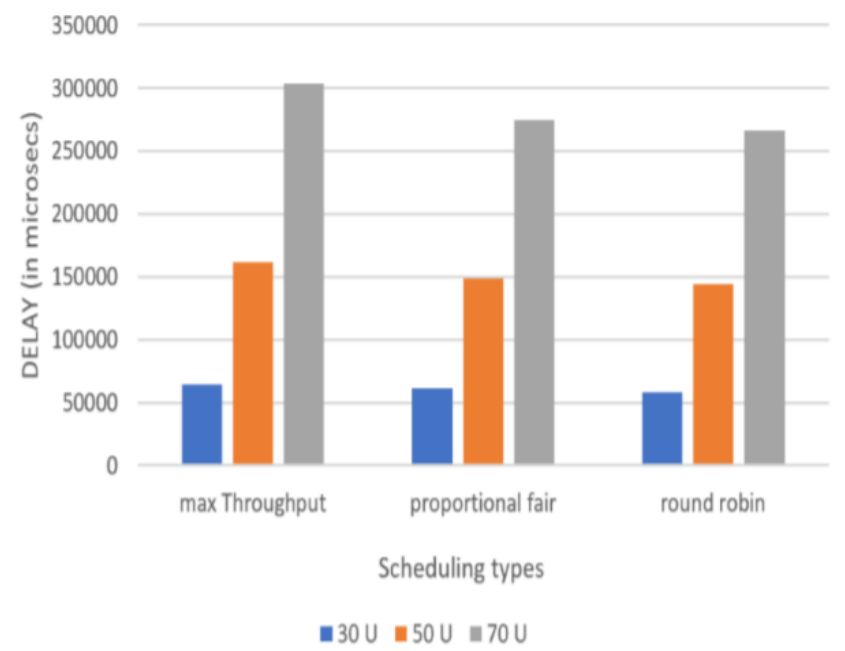

Figure 11: This histogram depicts the comparison of delay between the types different scheduling types for different no. of users in a LTE network

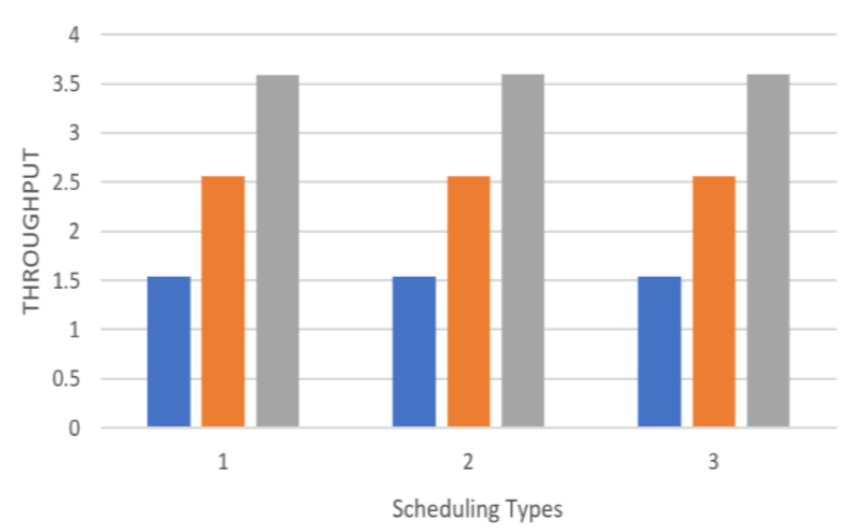

Figure 12: This figure depicts how the throughput varies with different scheduling types, where 1 is maximum throughput, 2 is proportional fair and 3 is round robin 
Table 2: Delay vs no. of users for different scheduling types

\begin{tabular}{|l|l|l|l|}
\hline Scheduling Type & 30 & 50 & 70 \\
\hline max throughput & 64132.03 & 161465.7 & 303208.8 \\
\hline proportional fair & 61335.5 & 148913.9 & 274285.8 \\
\hline Round robin & 58446.82 & 144211.1 & 265918.3 \\
\hline
\end{tabular}

Table 3: Throughput vs no. of users for different scheduling types

\begin{tabular}{|l|l|l|l|}
\hline \multicolumn{1}{|c|}{ Users } & 30 & 50 & 70 \\
\hline max throughput & 1.539781 & 2.561884 & 3.591695 \\
\hline proportional fair & 1.537753 & 2.560896 & 3.593733 \\
\hline Round robin & 1.537878 & 2.563015 & 3.594647 \\
\hline
\end{tabular}

Table 4: Spectral efficiency vs no. of users

\begin{tabular}{|l|l|}
\hline USERS & SPECTRAL EFFICIANCY \\
\hline 3 & 0.0149732 \\
\hline 5 & 0.0251789 \\
\hline 10 & 0.0469426 \\
\hline 30 & 0.15 \\
\hline 50 & 0.128 \\
\hline
\end{tabular}

Table 5: Delay vs users

\begin{tabular}{|l|l|}
\hline Number of users & Delay $(\mu \mathrm{s})$ \\
\hline 3 & 3425.582 \\
\hline 5 & 4858.585 \\
\hline 10 & 15137.603 \\
\hline 30 & 115276.2317 \\
\hline 50 & 161465.7 \\
\hline
\end{tabular}

\section{CONCLUSION}

In this paper, we considered a LTE network on which we performed some parametric analysis. Firstly we observed a trend in spectral efficiency by altering number of users and got a maximum spectral efficiency of $15 \%$.Then we calculated the trend in delay of the system with increase in number of users and it shows that delay increases with number of users. Then we compared the 3 popular scheduling types, namely maximum throughput, proportional fair and round-robin. We first performed individual analysis of trends in throughput and delay of all the 3 scheduling types. Then we compared the throughput and delay of all the 3 scheduling types by varying the number of users. From the above histograms (figures 11 and 12) we can infer that round robin scheduling provides us with maximum throughput and minimum latency among all the 3 scheduling types. Hence from this experiment we can conclude that round-robin is the best scheduler that can be used in a LTE network.

\section{ACKNOWLEDGMENT}

We would like to express gratitude to VIT University and the organization fFor providing us with necessary equipment and valuable suggestions without which we couldn't complete this experiment. We would like to thank the people in charge of the lab for their insights to this project.

\section{REFERENCES}

1. Sternad, Mikael \& Svensson, Tommy \& Ottosson, Tony \& Ahlen, Anders \& Svensson, Arne \& Brunstrom, Anna. (2007). Towards Systems Beyond 3G Based on Adaptive OFDMA Transmission. Proceedings of the IEEE. 95. 2432 - 2455 10.1109/JPROC.2007.907119.

2. R. E. Ahmed and H. M. AlMuhallabi, "Throughput-fairness tradeoff in LTE uplink scheduling algorithms," 2016 International Conference on Industrial Informatics and Computer Systems (CIICS), Sharjah, 2016, pp. 1-4. doi: 10.1109/ICCSII.2016.7462415.

3. S. C. Nguyen, K. Sandrasegaran and F. M. J. Madani, "Modeling and simulation of packet scheduling in the downlink LTE-advanced," The 17th Asia Pacific Conference on Communications, Sabah, 2011, pp. 53-57.

doi: 10.1109/APCC.2011.6152759.

4. Caushaj, Eralda; Ivanov, Ivan; Fu, Huirong; Sethi, Ishwar; and Zhu, Ye, "Evaluating Throughput and Delay in $3 G$ and $4 G$ Mobile architectures" (2014). Electrical Engineering \& Computer Science Faculty Publications. 300.

5. Barayan, Yaser \& Kostanic, Ivica. (2013). Performance Evaluation of Proportional Fairness Scheduling in LTE. Lecture Notes in Engineering and Computer Science. 2. 712-717.

6. T. Bu, L. Li and R. Ramjee, "Generalized Proportional Fair Scheduling in Third Generation Wireless Data Networks," Proceedings IEEE INFOCOM 2006. 25TH IEEE International Conference on Computer Communications, Barcelona, 2006, pp. 1-12.

7. P. Vieira, P. Queluz and A. Rodrigues, "LTE spectral efficiency using spatial multiplexing MIMO for macro-cells," 2008 2nd International Conference on Signal Processing and Communication Systems, Gold Coast, QLD, 2008, pp. 1-6.5.

8. Ravikumar CV, Kalapraveen bagadi."Robust Neural Network based multiuser detector in MC-CDMA for multiple access mitigation", Indian Journal of Science \& Technology. Vol 9, issue 30, 2016

9. Ravikumar CV, Kalapraveen bagadi."Performance analysis of HSRP in provisioning layer-3 Gateway redundancy for corporate networks ”, Indian Journal of Science \& Technology. Vol 9, issue 20, 2016

10. Ravikumar CV, Kalapraveen bagadi."Performance analysis of ipv4 to ipv6 transition methods ", Indian Journal of Science \& Technology. Vol 9, issue 20, 2016

11. Ravikumar CV, Saranya KC. "Implementing mobile adhoc network with improved AODV protocol", International Journal of Applied Engineering and Research. Vol 11 no. 9, 2016.

\section{AUTHOR PROFILE}

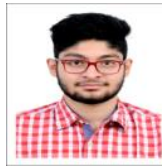

Rahul Varma bachelor's in technology, Electronics and Communication Engineering, VIT University.

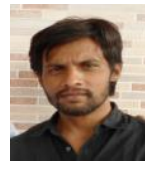

Ravi Kumar CV received M.Tech Degree in Digital Electronics and Communication Systems from JNTU Ananthapur, India in 2009. He received Ph.D degree in wireless communication and working as Assistant Professor in School of Electronics (SENSE), VIT University, Vellore, India in 2018. His areas of interest are wireless networking, MC-CDMA Artificial intelligence, soft computing techniques and neural networks 